\title{
Haemorrhoidal LASER Procedure (HeLP)- A painless Treatment for Haemorrhoid
}

\author{
ASMZ RAHMAN $^{\mathrm{a}}$, ASMT RAHMAN ${ }^{\mathrm{b}}$, M HASAN $^{\mathrm{c}}, \mathrm{S} \mathrm{CHANDRA}^{\mathrm{d}}$
}

Summary:

Introduction: According to the vascular theory arterial overflow in the superior haemorrhoidal arteries would lead to dilatation of the haemorrhoidal venous plexus. The technique helps to promote the obliteration of the terminals of the superior rectal artery. The objective of this study is to describe the results of 100 patients with hemorrhoidal disease treated by the Hemorrhoidal LASER Procedure technique.

Method: Under SAB anesthesia terminal arteriole of the upper rectal arteries were fulgurated by fiber laser diode, causing interruption of hemorrhoidal flow. This procedure is repeated circumferentially, following the clockwise positions. This procedure can be performed as a day care surgery.

Results: Between January 2018 to January 2019, 100 patients underwent the Hemorrhoidal LASER Procedure technique for hemorrhoidal disease grades I, II and III. Total 100 patients were treated with HeLP procedure. Among them number of men was 81 and female was 19.The procedure

\section{Introduction}

Hemorrhoids, also called piles are masses or clumps of tissues which consist of enlarged, bulging blood vessels and surrounding supporting tissues present in the anal canal of an individual. It is a condition characterized by the prolapsed of an anal cushion that may result in bleeding and pain. This condition is a common ailment among the adults. More than the men and women aged 50 years will experience hemorrhoid symptoms at least once during their life time. ${ }^{1}$ However, there have been

a. Prof. Dr. ASM ZAhidur Rahman, Head of Surgery Department, Shaheed Monsur Ali Medical College, Uttara

b. Dr. Tanjilur Rahman, Junior Consultant, Faridpur Medical College

c. Dr. Mehedi Hasan, Assistant Registrar (Surgery), Shaheed Monsur Ali Medical College, Uttara

d. Dr. Sushanta Chandra, Medical Officer (Surgery)

Address of Correspondence to: Prof. Dr. ASM ZAhidur Rahman,FCPS(Surgery),Head of Surgery Department,Shaheed Monsur Ali Medical College, Uttara,Dhaka. Email:asmrahman@gmail.com

Received: 12 March, 2019

Accepted: 1 November, 2019 proved to be successful at 3 month follow up of $92 \%$ patient. No major adverse effects or complications were reported. Post operative minor pain that required medication was 5 cases (5\% patient). Postoperative minor bleeding was observed in 3 cases that were managed conservatively. At 3 month after procedure resolution of symptoms were observed in $88 \%$ cases with overall satisfaction rate was $89 \%$. Significantly higher quality of life was observed in laser haemorrhoidal procedure.

Conclusion: Hemorrhoidal LASER Procedure is a painless outpatient technique.It is a safe, effective, improve symptoms \& chance of cure is high compared to other conventional surgery. Similar benefits are seen for patient with severe anal spasm, external thrombosis, fissure and sentinel tags $\&$ fistula.

Keywords: Hemorrhoids; Hemorrhoidal LASER procedure; Hemorrhoidal dearterialization, LHP.

(J Bangladesh Coll Phys Surg 2020; 38: 18-22) DOI: http://dx.doi.org/10.3329/jbcps.v38i1.44684

incidences where children and the elderly have also been diagnosed with this condition[2,3] Hemorrhoid disease is said to be the fourth leading outpatient gastrointestinal diagnosis, accounting for 3.3 million ambulatory care visits in the United States.[4] Although so common, only around $4 \%$ seek medical help.[5]

The treatment options for symptomatic haemorrhoid have varied over time that includes conservative medical management, non-surgical treatment and various surgical techniques. The various non-surgical treatments include rubber band ligation (RBL), injection sclerotherapy, cryotherapy, infrared coagulation, laser therapy and diathermy coagulation. In search for a nonsurgical technique for hemorrhoidal disease with less postoperative pain,Salfi et al [6] described the Hemorrhoidal LASER Procedure(HeLP) technique. It is a minimally-invasive technique with satisfactory results and less postoperative complications. This publication aims to describe 100 cases in which the hemorrhoid laser procedure was done. A three month follow-up was carried out, analyzing clinical and functional aspects of patients, evaluating pre- and postoperative symptoms 
of the studied population, description of the technique used, need for anesthesia for the procedure and the overall satisfaction index related to the technique.

\section{Material and Method}

Between January 2018 and January 2019, 100 patients were submitted to the HeLP procedure for hemorrhoidal disease grades I and II, or grade III with little mucosal prolapse. Grade IV and grade III hemorrhoids with large mucosal prolapse were excluded from the study. Data were prospectively collected and analyzed.

Before undergoing the surgical procedure, the patients were submitted to proctoscopy to exclude other possible causes of bleeding.Colonoscopy was done only those cases where sources of per-rectal bleeding was suspected other than haemorrhoid.

The patients signed the informed consent, which explained about the procedure to be performed. Anoscopy was performed to evaluate internal hemorrhoids after 30 days postoperatively. Quality of life assessment was questioned after the $1^{\text {st }}$ week, 1 month and 3 month post operatively

\section{HeLP Procedure}

This prospective study of 100 patients was performed in Shaheed Monsur Ali Medical College, Uttara \& Lubana General Hospital,Dhaka. After a detailed physical examination \& proctoscopy Laser procedure was performed with diode Lasotronix Laser. The patient is placed in the lithotomy position, under SAB anesthesia. Antibiotic prophylaxis with ceftriaxon was used in all cases. A disposable proctoscope that was specially designed for the hemorrhoid laser procedure is placed in the patient's distal rectum and anal canal.

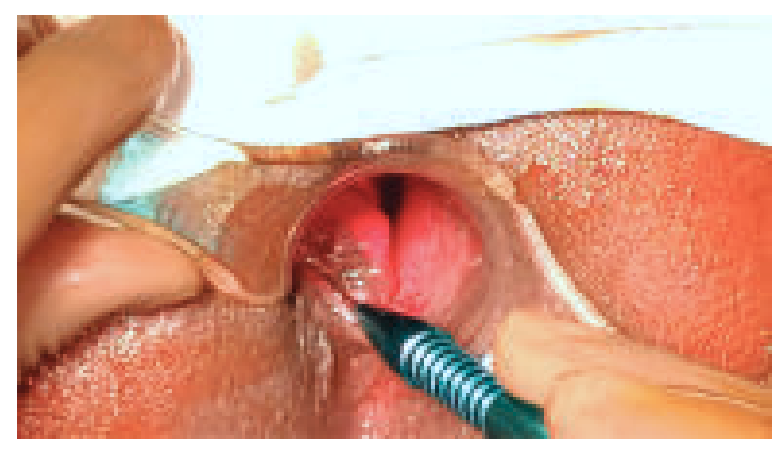

Fig.-1: Laser-diode device used in our procedure was calibrated with a power of $8-10 \mathrm{~W}$
The Laser shots were delivered with a 980 diode through a $600 \mathrm{~nm}$ optical laser fiber in a continuous fashion. The depth of shrinkage was regulated by the power \& duration by the laser beam. (Figure $1 \& 2$ ). The Laserdiode device used in our study was calibrated with a power of 8-10 W. Interruption of the artery supplying blood flow is performed by activating LASER energy through the optical fiber. First, two operator-controlled shots are performed, at $2 \mathrm{~mm}$ from the mucosa, to promote scorching of the latter, followed by three preset shots for arterial obliteration. Each shot lasts $1.2 \mathrm{~s}$, with a $0.6 \mathrm{~s}$ interval between them.

A clockwise rotation of the proctoscope allows application of laser to all branches of the superior rectal artery. In this way, the position of the clock hours is followed, checking at 1, 2, 3 O'clock and so on. A maximum of 12 branches can be treated at the same surgical procedure.

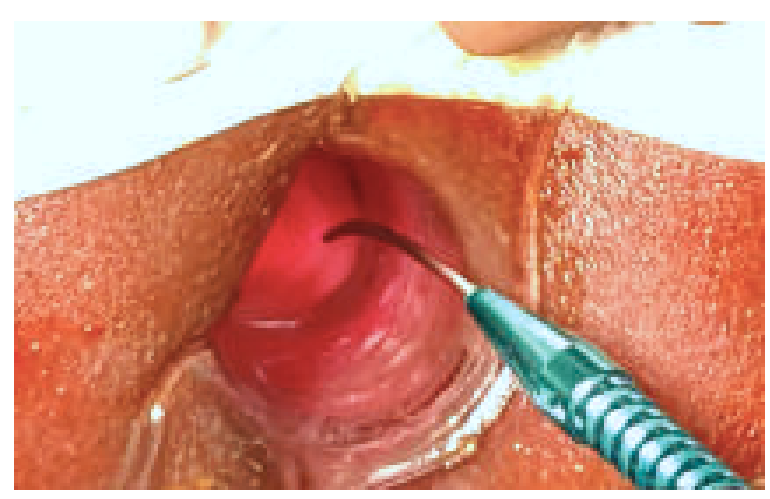

Fig.-2: Laser-diode device used in our procedure was calibrated with a power of 8-10 W

HeLP procedure was performed as an outpatient procedure. No bowel preparation was required. Patient was instructed not to take residual diet like meat \& vegetables 2 days before laser procedure. Patients were discharged from the hospital within 4 to 6 hours and were followed for 2 to 6 months after procedure. The patients were followed for post-operative pain, recovery $\&$ assessment of haemorrhoidal symptoms. Postoperative pain was assessed by Visual Analogue Scale (VAS) after 1 week, 2 week and after 1 month.

The laser hemorrhoidectomy was applied to 100 patients, 19 women and 81 men, with a mean age of 36 years ( 6 year -65 years). Population submitted to surgery having 
$80 \%$ hemorrhoids grades I and II and $20 \%$ of the sample had grade III hemorrhoidal disease.

Regarding the surgical indication, $77 \%$ patients had hematochezia as the main complaint treated clinically, $15 \%$ patients had anal discomfort secondary to prolapse during evacuation and $8 \%$ patients complained of anal pruritus (Table 1).

Table-I

Patients demography and baseline characteristics

Characteristics

Mean age (years)

Gender (\%)

\begin{tabular}{lc}
\hline Male & $81 \%$ \\
Female & $19 \%$ \\
Hemorrhoid grade (\%) & \\
Grade I \& II & $80 \%$ \\
Grade III & $20 \%$ \\
Main symptoms (\%) & \\
Bleeding & $77 \%$ \\
Anal discomfort & $15 \%$ \\
Pruritus & $8 \%$ \\
Time of surgery(in minute) & $10(7-13)$ \\
\hline
\end{tabular}

The mean surgical time was $10 \mathrm{~min}$, ranging from 7 to 13 min (Table 1). Only 2 patients were anxious in the preoperative period. Bleeding during the surgical procedure occurred in 3 patients, but all were treated with LASER fiber, requiring no more invasive maneuvers for hemostasis. (Table 3)

The mean time between surgery and hospital discharge was 4 hour. At the re-evaluation on day 7 postoperatively, 15 patients complained of self-limited bleeding and 8 patients complained of tenesmus, which showed complete resolution during follow-up.

On day 30 postoperatively, 81 of the 100 patients had a reduction of grade in the classification of hemorrhoidal disease The bleeding decreased in all cases for which it was the main complaint. At 3 months postoperatively, 88 of 100 patients reported symptom disappearance.

The overall satisfaction with the surgical and postoperative procedures reached $89 \%$, and $81 \%$ of the patients showed reduction of grade of hemorrhoidal disease that has been assessed by proctoscopy at follow up (Table $3 \& 4$ ).
Table-II: Main symptoms of study population

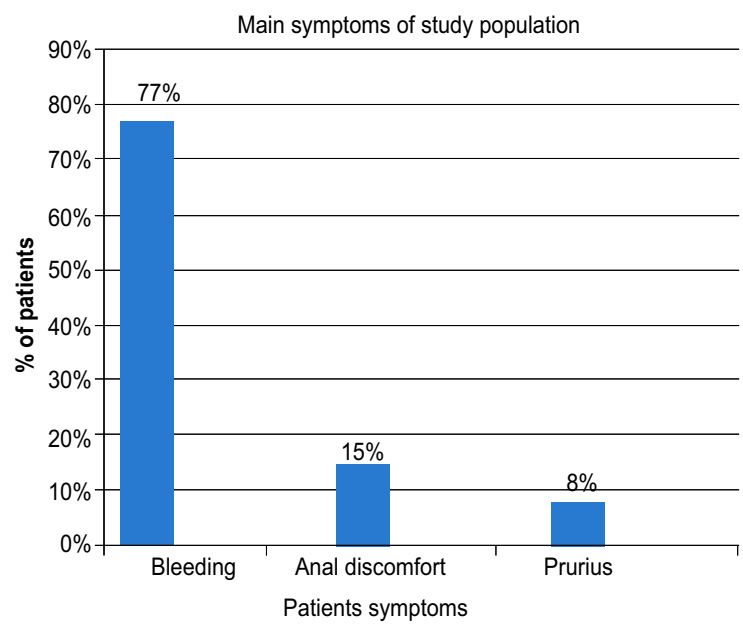

Chart 1: Grading of Haemorrhoid of study population

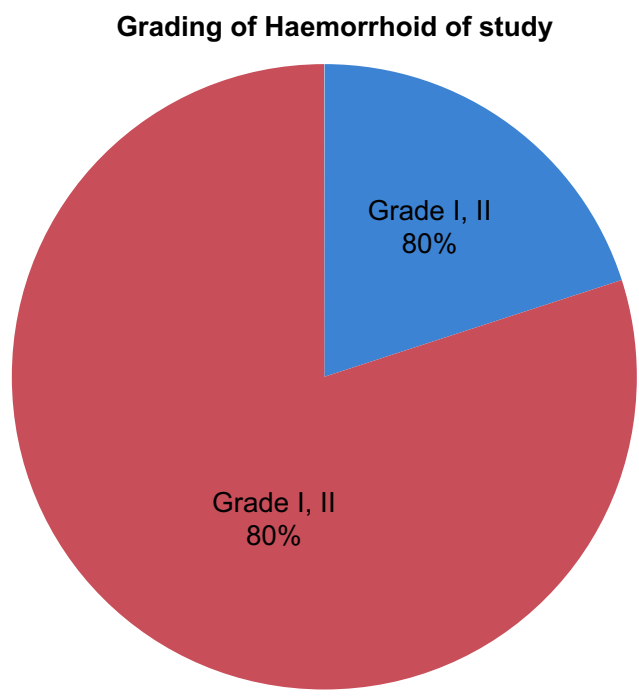

Table-III

Perioperative and follow-up period.

Patients demography and Patients $(n=100)$ baseline characteristics

Perioperative bleeding (\%) $3 \%$

Postoperative tenesmus (\%) $15 \%$

Evaluation at 3 months (\%)

Symptoms disappearance $88 \%$

Overall satisfaction index $89 \%$

Haemorrhoids volume reduction $81 \%$ 


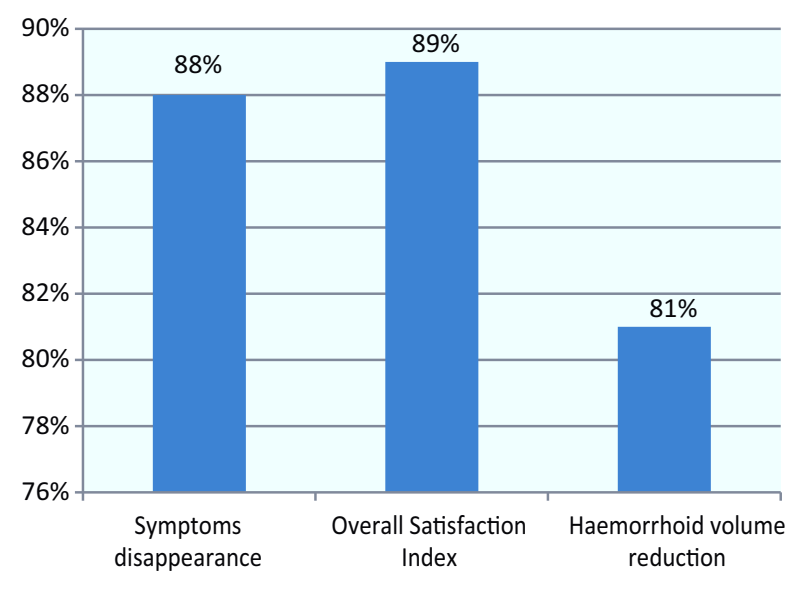

Evaluation of system after 3 months

Fig.-4: Evaluation at 3 months (\%)

\section{Discussion}

The need for treatment of haemorrhoid is primarily based on subjective perception of severity of symptoms \& assignment of treatment is decided on the traditional classification of haemorrhoid.[7]

Multiplicity of treatment modality has added confusion in decision about the treatment method. The question of the optimal treatment technique remain unanswered.

The conventional techniques bring fear to many patients who refrain from being adequately treated, either due to previous experience, or from reports of friends and relatives. In a study of the University of SaoPaolo, Brazil, they stated that Laser Haemorrhoidectomy had the advantages of being haemostatic, bactericidal, fast healing, not affecting neighboring structures, less postoperative complications \& less haemorrhage \& stenosis[8] .

Current techniques aim to interrupt the flow in the terminal portion of the arterioles, with no tissue excision and, consequently, less painful sensation when compared to the excisional or conventional techniques. $[9,10,11]$

The HeLP technique is well-established for the treatment of grades I and II and grade III. It has been successfully disseminated in the United Kingdom, Italy, Czech Republic, Spain, and Turkey, as well as in Mexico, Argentina and others. [12] Despite our experience described in this study, new publications with larger samples and longer follow-up are necessary in the treatment of these diseases.
It is noteworthy the fact that it is a technique that can be repeated several times, as many as necessary, without causing damage to the anorectal anatomy and physiology. The fact that it does not require anesthesia does not prevent its performance with the aid of sedation or even compound analgesia. [12-17]

Our study was limited to hemorrhoids grades I and II, as well as grade III hemorrhoids with small prolapse, but new studies have demonstrated good results of HeLP use with associated mucopexy when the patient has a large mucosal prolapse. [18]

Post operative pain is the most important complication that disturbs our patients and makes them reluctant to surgery. In spite of many modifications made to the haemorrhoidal surgical techniques, post operative pain and discomfort remain the major concerns.[19]It is reported that the application of Laser technique in the treatment of haemorrhoids was safe, effective and painless.[20-21[Our study showed that HeLP is safe procedure associated with less postoperative pain.

The same study shown that Laser haemorrhoidectomy is associated with lesser duration of time compared with open haemorrhoidectomy. [20-21] Our study have similar results of less operative time of average 10 minutes

There are also some economic barriers, mainly because it is a relatively new and less disseminated technique in our country, but comparative studies with other existing techniques have reported a higher index of patient satisfaction [12-17]. This procedure decreases the costs of the hospital stay, which represents an important part of the amount spent with treatment.

Several studies compared the efficacy of various haemorrhoidal laser treatment to rubber banding and to existing common surgery procedures as Milligan Morgan,ligature removal etc. All of these studies indicate that laser techniques presents similar or even better results and may present a safe and effective alternative approach in haemorrhoidal disease treatment.post operative infection have not been reported. The perioperative bleeding is minimal, there is no postoperative urinary retention and need for postoperative analgesia is significantly decreased. The main advantages of this minimally invasive treatment for haemorrhoidal disease are the significant decrease on postoperative pain intensity and duration and they 
minimize the possibility of anal sphincter damage with subsequent anal incontinence developement. A possible disadvantage is the need of single use of laser fiber that may increase the cost of treatment. [22,23,24]

\section{Conclusion}

We conclude that Haemorrhoidal Laser Procedure (HeLP) is a safe, painless, ambulatory, and easy-toperform technique, in which the learning curve becomes small can be repeated the same procedure if performed in a single patient, although there are still enough to be clarified.

more perspectives well organised studies with longer follow up are required to demonstrate the exact advantages of this technique and especially to define the recurrence rate.

\section{References:}

1. Bailey HR. Innovations for age-old problem: hemorrhoids in the female patient. Female Patient. 2004;29:17-23.

2. Heaton ND, Davenport M, Howard ER. Symptomatic hemorrhoids and anorectal varices in children with portal hypertension. J Pediatr Surg.1992;27(7):833-5.

3. Navarra L, Pietroletti R, Maggi G, Leardi S, Simi M. Diagnosis and treatment of haemorrhoids in the eldery: results from 291 patients. Techniques in Coloproctology. 2000;3(3):127-30

4. Everhart JE, Ruhl CE. Burden of digestive diseases in the United States part I: overall and upper gastrointestinal diseases. Gastroenterol. 2009;136(2):376-86.

5. Liebach JR, Cerda JJ. Hemorrhoids: modern treatment methods. Hosp Med. 1991;53:68

6. Salfi R (2009) A new technique for ambulatory hemorrhoidal treatment. Coloproctol 31: 99-103

7. Goligher J, Duthie H, Nixon H. London: Baillière Tindall; 1984. Surgery of the Anus Rectum and Colon.

8. Laurie Barcly. Best option for evaluating and treating hemorrhoids. BMJ. 2008 Feb 25;336:380-383.

9. Ratto C, Donisi L, Parello A, Litta F, Doglietto GB (2010) Evaluation of transanal hemorrhoidal dearterialization as a minimally invasive therapeutic approach to hemorrhoids. Dis Colon Rectum 53: 803-811.

10. Morinaga K, Hasuda K, Ikeda T (1995) A novel therapy for internal hemorrhoids: ligation of the hemorrhoidal artery with a newly devised instrument (Moricorn) in conjunction with a Doppler flowmeter. Am J Gastroenterol 90: 610-613.

11. Longo A (1998) Treatment of haemorrhoids disease by reduction of mucosa and haemorrhoidal prolapse with a circular suturing device: a new procedure, In: Proceedings of the Sixth World Congress of Endoscopic Surgery. Monduzzi 1998: 777-784.
12. Giamundo P, Cecchetti W, Esercizio L, Fantino G, Geraci G, et al. (2011) Doppler-guided Hemorrhoidal LASER Procedure for the treatment of symptomatic hemorrhoids: experimental background and short-term clinical results of a new mini-invasive treatment. Surg Endosc 25: 13691375 .

13. Sohn N, Aronoff JS, Cohen FS, Weinstein MA (2001) Transanal hemorrhoidal dearterialization is an alternative to operative hemorrhoidectomy. Am J Surg 182: 515-519.

14. Sobrado CW, Hora JA (2012) Transanal desarterialization guided by Doppler associated to anorectal repair in hemorrhoids: THD technic. ABCD Arq Bras Cir Dig 3: 19326.

15. Ramírez JM, Aguilella V, Elía M, Gracia JA, Martínez M (2005) Doppler-guided hemorrhoidal artery ligation in the management of symptomatic hemorrhoids. Rev Esp Enferm Dig 97: 97-103.

16. Qin PP, Huang B, Cai HJ, Ge Q, Wang ZL (2010) A prospective controlled comparative study of Dopplerguided hemorrhoidal artery ligation versus operative procedures for prolapse and hemorrhoids. Zhonghua $\mathrm{Yi}$ Xue Za Zhi 90: 3131-3134.

17. Pol RA, van der Zwet WC, Hoornenborg D, Makkinga B, Kaijser M, et al. (2010) Results of 244 consecutive patients with hemorrhoids treated with Doppler-guided hemorrhoidal artery ligation. Dig Surg 27: 279-284.

18. Giamundo P, Geraci M, Tibaldi L, Esercizio L, Agostini S, et al. (2014) Laser haemorrhoidal arterial closure (HeLP) with recto-anal-repair (RAR): a novel procedure for the treatment of advanced haemorrhoidal disease. Colorectal Dis 16: 21-36.

19. Yeo D, Tan KY, Haemorrhoidectomy - Making sense of surgical options. World J Gastroenterol. 2014;20:1697616983

20. Giamundo P, Salfi R, Geraci M, et al. The haemorrhoid laser procedure technique vs. rubber band ligation: a randomized trail comparing 2 mini invasive treatment for second and third degree haemorrhoids. Dis Colon Rectum.2011;54:693-698

21. Crea N, Pata G, Lippa M,et al. Haemorrhoid laser procedure: short and long term result from a prospective study.Am J Surg. 2014;208:21-25

22. De Nardi P, Tamburini AM, Gazzetta PG, Lemma M, Pascariola A, Asteria CR.Haemorrhoid laser procedure for second and third degree haemorrhoids: Result from a multicenter prospective study. Tech coloproctol 2016 Jul;20(7):455-9

23. Crea N, Pata G, Lippa M,Chiesa D, GregoriniME,Gandolfi P. Haemorrhoidal Laser Procedure: Short-and long term result from a prospective study.Am J Surgery 2014 Jul;208(1): 21-25

24. Malohu H, Gasi Z, Lazovic R, Islami H, Juniku- Shikololli A. Laser Haemorrhoidoplasty Procedure VS Open surgical haemorrhoidectomy: A trial comparing 2 treatments for haemorrhoid of third and fourth degree. Acta Inform Med 2014 Dec;22(6):365-7 